九州大学学術情報リポジトリ

Kyushu University Institutional Repository

\title{
ORTHOGONALITY OF HYPOTHESE AND IDENTIFIABILITY CONSTRAINTS IN THE ANALYSIS OF VARIANCE MODEL
}

Washio, Yasutoshi

Faculty of Science and Technology, Keio University

https://doi.org/10.5109/13375

出版情報: Bulletin of informatics and cybernetics. 22 (1/2), pp.39-54, 1986-03. Research Association of Statistical Sciences

バージョン :

権利関係 : 


\title{
ORTHOGONALITY OF HYPOTHESES AND IDENTIFIABILITY CONSTRAINTS IN THE ANALYSIS OF VARIANCE MODEL
}

By

\author{
Yasutoshi WASHIO*
}

\begin{abstract}
We consider a sequence of hypotheses $H_{i}: \boldsymbol{\beta}_{i}=\boldsymbol{O}, i=1,2, \cdots, k$, in a liner statistical model $\boldsymbol{y}=X \boldsymbol{\beta}+\boldsymbol{e}=X_{1} \boldsymbol{\beta}_{1}+X_{2} \boldsymbol{\beta}_{2}+\cdots+X_{k} \boldsymbol{\beta}_{k}+\boldsymbol{e}$. We assume that $X$ is not of full rank -in the analysis of variance modelsuitable identifiability constraints $B \boldsymbol{\beta}=\boldsymbol{O}$ are given in the model.

First, we give some results on the orthogonality of hypotheses by introducing the null space representation for the hypotheses. Then, we confine our model to the analysis of variance model and discuss the relationship between orthogonality of hypotheses and identifiability constraints. A method for finding the identifiability constraints which make all the considerable hypotheses orthogonal is proposed.
\end{abstract}

\section{Introduction}

Suppose we have a linear statistical model

$$
\begin{aligned}
\boldsymbol{y} & =X \boldsymbol{\beta}+\boldsymbol{e} \\
& =\left[\begin{array}{llll}
X_{1} & X_{2} & \cdots & X_{k}
\end{array}\right]\left[\begin{array}{c}
\boldsymbol{\beta}_{1} \\
\boldsymbol{\beta}_{2} \\
\vdots \\
\boldsymbol{\beta}_{k}
\end{array}\right]+\boldsymbol{e},
\end{aligned}
$$

where $\boldsymbol{y}$ is an $n \times 1$ vector of observation, $X$ is a known matrix of rank $r(r \leqq p)$ and called a design matrix, $\boldsymbol{\beta}$ is a $\not \times 1$ vector of unknown parameters, $X$ and $\boldsymbol{\beta}$ are expressed by partitioned matrices with appropriate sizes in the last formula in (1.1) and $\boldsymbol{e}$ is an $n \times 1$ vector of errors distributed as $N\left(0, \sigma^{2} I_{n}\right)$, where $\sigma^{2}$ is an unknown positive constant and $I_{n}$ is the $n \times n$ identity matrix.

The concept of orthogonality of hypotheses is mainly associated with the analysis of variance model. In the analysis of variance model, the design matrix $X$ is not of full rank and a set of linear constraints among parameters is usually introduced as a set of identifiability constraints to identify the parameters. Then, when $X$ is not of full rank, we assume that suitable identifiability constraints

$$
B \boldsymbol{\beta}=\mathbf{0}
$$

* Faculty of Science and Technology, Keio University. 14-1, Hiyoshi, 3 Chome, Kohokuku, Yokohama 223, Japan. 
are given in the model (1.1). A set of linear constraints $B \boldsymbol{\beta}=\mathbf{0}$ is called a set of identifiability constraints if the matrix $B$ satisfies the following conditions (i) and (ii): (i) The composite matrix $\left[\begin{array}{c}X \\ B\end{array}\right]$ has rank $p$.

(ii) No linear combination of the rows of $B$ is a linear combination of the rows of $X$ except the zero vector.

Let's denote the expectation of $\boldsymbol{y}, E(\boldsymbol{y})$, by $\boldsymbol{\theta}$. For any matrix $A$, the range space of $A$ will be denoted by $\Re[A]$, i. e. $\Re[A]=\{\boldsymbol{v} \mid \boldsymbol{v}=A \boldsymbol{u}$, for some $\boldsymbol{u}\}$ and the null space of $A$ will be denoted by $\mathfrak{R}[A]$, i. e. $\mathfrak{R}[A]=\{\boldsymbol{v} \mid A \boldsymbol{v}=\mathbf{0}\}$. For a subspace $\omega$ the orthogonal complement of $\omega$ will be denoted by $\omega^{\perp}$.

In the linear statistical model (1.1), we are interested in testing a sequence of linear hypotheses

$$
H_{i}: \boldsymbol{\beta}_{i}=\mathbf{0}, \quad i=1,2, \cdots, k .
$$

The hypotheses $H_{i}, i=1,2, \cdots, k$, can be expressed as follows: The linear model (1.1) states

$$
\theta \in \Omega
$$

and the hypotheses $H_{i}$ further states

$$
H_{i}: \boldsymbol{\theta} \in \omega_{i} \quad\left(\omega_{i} \subset \Omega\right),
$$

$i=1,2, \cdots, k$, where $\Omega=\mathfrak{R}[X], \omega_{i}=\mathfrak{R}\left[X_{(i)}\right]$ when $X$ is of full rank and $\omega_{i}=\{\boldsymbol{v} \mid \boldsymbol{v}=$ $\left.X_{(i)} \boldsymbol{\beta}_{(i)}, B_{(i)} \boldsymbol{\beta}_{(i)}=\mathbf{0}\right\}$ when $X$ is not of full rank, where $X_{(i)}$ is the matrix $X$ with the submatrix $X_{i}$ deleted, $B_{(i)}$ and $\boldsymbol{\beta}_{(i)}$ are similarly defined.

As to the orthogonality of hypotheses, there are two kinds of definitions: One is given by Kempthorne [3] and the other is given by Darroch-Silvey [1]. Although both of the definitions are given in a sense that all the considerable hypotheses $H_{1}, H_{2}, \cdots, H_{k}$ are orthogonal, we shall give their definitions in a slight different form here.

DEFINITION 1.1. (Kempthorne [3]) The two hypotheses $H_{i}$ and $H_{j}$ are said to be orthogonal $\left(H_{i} \perp H_{j}\right)$ if $\hat{\boldsymbol{\beta}}_{i}$ and $\hat{\boldsymbol{\beta}}_{j}$ are uncorrelated, i. e. if $\operatorname{cov}\left(\hat{\boldsymbol{\beta}}_{i}, \hat{\boldsymbol{\beta}}_{j}\right)=0$, where $\hat{\boldsymbol{\beta}}_{i}$ and $\hat{\boldsymbol{\beta}}_{j}$ denote the least squares estimates of $\boldsymbol{\beta}_{i}$ and $\boldsymbol{\beta}_{j}$ under the model (1.1). For hypotheses of more than two, they are said to be orthogonal if any two hypotheses of them are orthogonal.

Definition 1.2 (Darroch-Silvey [1]) The two hypotheses $H_{i}$ and $H_{j}$ are said to be orthogonal $\left(H_{i} \perp H_{j}\right)$ if the subspaces $\Omega, \omega_{i}, \omega_{j}$ satisfy the condition $\omega_{i}^{\frac{1}{2}} \Omega \perp \omega_{j}^{\frac{1}{j}} \cap \Omega$, i. e. if the orthogonal complements of $\omega_{i}$ and $\omega_{j}$ with respect to $\Omega$ are perpendicular. The definition of orthogonality of more than two hypotheses is the same as that in Definition 1.1 .

The equivalence between definitions 1.1 and 1.2 is proved by Seber [5].

Although the matrix $B$ in identifiability constraints (1.2) must satisfy some conditions, there still exist so many choices for $B$ and we may choose any of them in the theoretical point of view (see Scheffé [4]). In testing hypotheses, however, the choice for $B$ will alter orthogonality property of hypotheses. It is desirable to choose the matrix $B$ so that the number of hypotheses which are orthogonal is as many as possible, because the orthogonality of hypotheses makes the computation of the sums of squares for $H_{i}$, $i=1,2, \cdots, k$, easier (See Section 3). 
Seber [5] gave a necessary and sufficient condition on $B$ for all considerable hypotheses in two-way layouts with and without replications to be orthogonal by the different methods under some further restrictions on $B$.

It seems to the author that there still needs a slight general and simple method to check orthogonality of hypotheses under the given identifiability constraints and to select suitable identifiability constraints for orthogonality property. This paper is motivated by this question.

In Section 2, first, the null space representation for the hypotheses are introduced and some properties of matrices being used in the representation are given as lemmas. Then, we shall give some theorems on orthogonality of hypotheses, which will be used in Section 3. Lastly, we shall give simpler proofs of two well known results on orthogonality of hypotheses.

In Section 3, we shall confine our model to the analysis of variance model, and the relationship between identifiability constraints and orthogonality of hypotheses will be considered. A theorem which proposes a method for finding the identifiability constraints which make all the hypotheses orthogonal is given. The method is illustrated by some examples.

\section{Some Theorems on Orthogonality of Linear Hypotheses in the Linear Model}

First, we shall give the null space representation for the hypotheses $H_{1}, H_{2}, \cdots, H_{k}$. The linear model (1.1) states the expected value of $\boldsymbol{y}, \boldsymbol{\theta}$, belongs to $\Omega$.

When $X$ is of full rank, $\beta$ can be expressed as

$$
\boldsymbol{\beta}=\left(X^{\prime} X\right)^{-1} X^{\prime} \boldsymbol{\theta}
$$

by using $\boldsymbol{\theta}$ since $\boldsymbol{\theta}=X \boldsymbol{\beta}$, where the prime denotes the transpose of a matrix. If we put $A \equiv\left(X^{\prime} X\right)^{-1} X^{\prime}$ and if we partition $A$ into $k$ submatrices such as $A=\left[\begin{array}{c}A_{1} \\ \vdots \\ \vdots \\ A_{k}\end{array}\right]$ corresponding to the partition of $\beta,(2.1)$ turns out to be

$$
\boldsymbol{\beta}=A \boldsymbol{\theta} \text { or }\left[\begin{array}{c}
\boldsymbol{\beta}_{1} \\
\vdots \\
\boldsymbol{\beta}_{k}
\end{array}\right]=\left[\begin{array}{c}
A_{1} \boldsymbol{\theta} \\
\vdots \\
\vdots \\
A_{k} \boldsymbol{\theta}
\end{array}\right] \text {. }
$$

It is clear from the definition of $A, A_{1}, \cdots, A_{k}$ that

$$
\mathfrak{R}\left[A^{\prime}\right] \subset \Omega, \mathfrak{R}\left[A_{1}^{\prime}\right] \subset \Omega, \cdots, \mathfrak{R}\left[A_{k}^{\prime}\right] \subset \Omega .
$$

By making use of matrices $A_{1}, \cdots, A_{k}$, the hypotheses $H_{1}, \cdots, H_{k}$ can be written as

$$
H_{i}: \boldsymbol{\theta} \in \Omega \text { and } A_{i} \boldsymbol{\theta}=\mathbf{0},
$$

$i=1,2, \cdots, k$.

When $X$ is not of full rank, as mentioned in Section 1 , suitable identifiability constraints $B \boldsymbol{\beta}=\mathbf{0}$ are given to identify the parameters, where $B$ is a $(p-r) \times p$ matrix of rank $p-r$. From the property of the matrix $B$, the equation

$$
\left[\begin{array}{c}
X \\
B
\end{array}\right] \boldsymbol{\beta}=\left[\begin{array}{c}
\boldsymbol{\theta} \\
\mathbf{0}
\end{array}\right]
$$


has a unique solution in $\boldsymbol{\beta}$ for every $\boldsymbol{\theta} \in \Omega$. Since $\left[\frac{X}{B}\right] \equiv G$ has rank $p$, from (2.5), we have

$$
\boldsymbol{\beta}=\left(G^{\prime} G\right)^{-1} G^{\prime}\left[\begin{array}{c}
\boldsymbol{\theta} \\
\hdashline \\
\mathbf{0}
\end{array}\right]=\left(G^{\prime} G\right)^{-1} X^{\prime} \boldsymbol{\theta}
$$

For this case, if we put $A \equiv\left(G^{\prime} G\right)^{-1} X^{\prime}$ and if $A$ is partitioned into $k$ submatrices as before, then $\boldsymbol{\beta}$ can again be expressed as (2.2). Furthermore, the property (2.3) also holds true, and the hypotheses $H_{1}, H_{2}, \cdots, H_{k}$ can be expressed as (2.4).

Therefore, in general, in the linear model (1.1) we can express $\beta$ as

$$
\boldsymbol{\beta}=A \boldsymbol{\theta} \text { or }\left[\begin{array}{c}
\boldsymbol{\beta}_{1} \\
\vdots \\
\vdots \\
\boldsymbol{\beta}_{k}
\end{array}\right]=\left[\begin{array}{c}
A_{1} \boldsymbol{\theta} \\
\vdots \\
\hdashline A_{k} \boldsymbol{\theta}
\end{array}\right]
$$

and the hypotheses $H_{i}, i=1,2, \cdots, k$, can be expressed as

$$
H_{i}: \boldsymbol{\theta} \in \Omega \text { and } A_{i} \boldsymbol{\theta}=\mathbf{0},
$$

where $A, A_{1}, \cdots, A_{k}$ have the property such that

$$
\mathfrak{R}\left[A^{\prime}\right] \subset \Omega, \quad \Re\left[A_{i}^{\prime}\right] \subset \Omega, \quad i=1,2, \cdots, k .
$$

Let $C$ be a matrix such that $\Omega=\Re[C]$ and the rows of $C$ are linearly independent. The linear model (1.1) says

and the hypothesis $H_{i}$ states

$$
\boldsymbol{\theta} \in \Omega=\mathfrak{R}[C]
$$

$$
H_{i}: \boldsymbol{\theta} \in \boldsymbol{\omega}_{i}=\mathfrak{\Re}\left[\begin{array}{c}
C \\
A_{i}
\end{array}\right]
$$

Here we note that

$$
\Re\left[A_{i}^{\prime}\right] \perp \Re\left[C^{\prime}\right], \quad i=1,2, \cdots, k
$$

from (2.9) and the definition of $C . \quad(2.10)$ and (2.11) are the null space representations for the model (1.1) and the hypothesis $H_{i}$. The properties of (2.9) and (2.12) for the matrices $A, A_{i}, C$ in the null space representation are important and will often be used later on.

LEMMA 2.1. In the null space representation for the linear model and the hypothesis $H_{i}$, we have

PROOF.

$$
\omega_{i}^{\perp} \cap \Omega=\Re\left[A_{i}^{\prime}\right]
$$

$$
\begin{aligned}
\Omega \cap \omega_{i}^{1} & =\mathfrak{R}[C] \cap\left(\Re\left[\begin{array}{c}
C \\
A_{i}
\end{array}\right]\right)^{\perp} \\
& =\mathfrak{R}[C] \cap \Re\left[C^{\prime}: A_{i}^{\prime}\right] .
\end{aligned}
$$

We show (2.13) is equal to $\Re\left[A_{i}^{\prime}\right]$. It is clear from (2.9) that

$$
\mathfrak{R}[C] \cap \Re\left[C^{\prime}: A_{i}^{\prime}\right] \supset \mathfrak{R}\left[A_{i}^{\prime}\right] .
$$

If $\boldsymbol{x}$ belongs to (2.13), then $C \boldsymbol{x}=\mathbf{0}$ and $\boldsymbol{x}=C^{\prime} \boldsymbol{a}+A_{i}^{\prime} \boldsymbol{\gamma}$ for some $\boldsymbol{a}$ and $\boldsymbol{\gamma}$. Thus 


$$
\begin{aligned}
C\left(C^{\prime} \boldsymbol{\alpha}+A_{i}^{\prime} \boldsymbol{\gamma}\right) & =C C^{\prime} \boldsymbol{\alpha}+C A_{i}^{\prime} \boldsymbol{\gamma} \\
& =C C^{\prime} \boldsymbol{\alpha}+\mathbf{0}=\mathbf{0} .
\end{aligned}
$$

Since $C C^{\prime}$ has the inverse matrix, we have $\boldsymbol{\alpha}=\mathbf{0}$ from the above equation. Therefore, $\boldsymbol{x}=A_{i}^{\prime} \boldsymbol{\gamma} \in \mathfrak{R}\left[A_{i}^{\prime}\right]$ and the lemma is proved.

LEMMA 2.2. In the null space representation for the linear model and the hypotheses $H_{i}, \quad i=1,2, \cdots, k$,

$$
H_{i} \perp H_{j} \Leftrightarrow \Re\left[A_{i}^{\prime}\right] \perp \Re\left[A_{j}^{\prime}\right] .
$$

Proof. The lemma follows from Definition 1.2 and Lemma 2.1. q. e. d.

LEMMA 2.3. In the null space representation for the linear model and the hypotheses $H_{i}, i=1,2, \cdots, k$, the equation

holds true if and only if

$$
\mathfrak{N}\left[\begin{array}{c}
C \\
A_{j_{1}} \\
\vdots \vdots \\
\hdashline A_{j_{q}}
\end{array}\right] \cap\left(\left[\begin{array}{c}
C \\
\hdashline A_{j_{1}} \\
\vdots \vdots \\
\hdashline A_{j_{q}} \\
A_{l}
\end{array}\right]\right)^{\perp}=\mathfrak{R}\left[A_{l}^{\prime}\right]
$$

$$
H_{l} \perp H_{j_{1}}, H_{l} \perp H_{j_{2}}, \cdots, H_{l} \perp H_{j_{q}} .
$$

Proof. Since the left hand side of the equation (2.14) is

$$
\begin{aligned}
\mathfrak{N}\left[\begin{array}{c}
C \\
\hdashline A_{j_{1}} \\
\hdashline \vdots \\
\hdashline A_{j_{q}}
\end{array}\right] \cap\left(\left[\begin{array}{c}
C \\
\hdashline A_{j_{1}} \\
\vdots \vdots \\
\hdashline A_{j_{q}} \\
\hdashline A_{l}
\end{array}\right]\right) & =\mathfrak{N}\left[\begin{array}{c}
C \\
\hdashline A_{j_{1}} \\
\vdots \vdots \\
A_{j_{q}}
\end{array}\right] \cap \Re\left[C^{\prime} A_{j_{1}}^{\prime} \cdots A_{j_{q}}^{\prime} A_{l}^{\prime}\right] \\
& =\mathfrak{N}\left[\begin{array}{c}
C \\
\hdashline \bar{A}_{j}
\end{array}\right] \cap \Re\left[C^{\prime} \bar{A}_{j}^{\prime} A_{l}^{\prime}\right],
\end{aligned}
$$
where $\bar{A}_{j}$ is the matrix consisting of linearly independent rows of $\left[\begin{array}{c}A_{j_{1}} \\ \vdots \\ A_{j_{q}}\end{array}\right]$, it is clear by
Lemma 2.2 that (2.14) implies (2.15).

Conversely suppose that (2.15) is true. It is clear from (2.16) and Lemma 2.2 that

$$
\mathfrak{N}\left[\begin{array}{c}
C \\
\hdashline A_{j_{1}} \\
\vdots \\
A_{j_{q}}
\end{array}\right] \cap\left(\mathfrak{N}\left[\begin{array}{c}
C \\
\hdashline A_{j_{1}} \\
\vdots \\
A_{j_{g}} \\
\hdashline A_{l}
\end{array}\right]\right) \supset \mathfrak{N}\left[A_{l}^{\prime}\right] .
$$

If $\boldsymbol{x}$ belongs to the left-hand side of (2.14), then

$$
\boldsymbol{x} \in \mathfrak{N}\left[\begin{array}{c}
C \\
\bar{A}_{j}
\end{array}\right] \text { and } \boldsymbol{x}=C^{\prime} \boldsymbol{\alpha}+\bar{A}_{j}^{\prime} \boldsymbol{\gamma}+A_{l}^{\prime} \boldsymbol{\delta}
$$

for some $\boldsymbol{\alpha}, \boldsymbol{\gamma}$ and $\boldsymbol{\delta}$. From $C \boldsymbol{x}=\mathbf{0}$, we have $\boldsymbol{\alpha}=\mathbf{0}$ by using the property of matrices $C, \bar{A}_{j}^{\prime}, A_{l}^{\prime}$. Similarly, from $\bar{A}_{j} \boldsymbol{x}=\mathbf{0}$, we have $\boldsymbol{\gamma}=\mathbf{0}$ by using the assumption. Thus $\boldsymbol{x}=A_{l}^{\prime} \boldsymbol{\delta}$, which means $\boldsymbol{x} \in \mathfrak{R}\left[A_{l}^{\prime}\right]$. Then (2.14) holds true.

In this paper, the least squares estimates of $\boldsymbol{\beta}_{i}$ under various situations will be 
considered. The least squares estimate of $\boldsymbol{\beta}_{i}$ under the general model (1.1) will be denoted by $\hat{\boldsymbol{\beta}}_{i}$. The least squares estimate of $\boldsymbol{\beta}_{\boldsymbol{i}}$ under $\boldsymbol{\beta}_{j}=\mathbf{0}$, that is, under $H_{j}$, will be denoted by $\hat{\boldsymbol{\beta}}_{i, H_{j}}$. Similarly, we shall use the notations $\hat{\boldsymbol{\beta}}_{i, H_{j} \cap H_{l}}, \hat{\boldsymbol{\beta}}_{i, H_{j} \cap H_{l} \cap H_{m}}$, etc. For example, $\hat{\boldsymbol{\beta}}_{i, H_{j} \cap H_{l}}$ denotes the least squares estimate of $\boldsymbol{\beta}_{i}$ under $\boldsymbol{\beta}_{j}=\mathbf{0}$ and $\boldsymbol{\beta}_{l}=\mathbf{0}$.

THEOREM 2.1 .

$$
H_{i} \perp H_{j} \Leftrightarrow \hat{\boldsymbol{\beta}}_{i}=\hat{\boldsymbol{\beta}}_{i, H_{j}} \quad\left(\hat{\boldsymbol{\beta}}_{j}=\hat{\boldsymbol{\beta}}_{j, H_{i}}\right) .
$$

In words, $H_{i}$ and $H_{j}$ are orthogonal if and only if the least squares estimate of $\boldsymbol{\beta}_{i}$ $\left(\boldsymbol{\beta}_{j}\right)$ is the same whether or not we put $\boldsymbol{\beta}_{j}=\mathbf{0}\left(\boldsymbol{\beta}_{i}=\mathbf{0}\right)$.

PROOF. Since $\boldsymbol{\beta}_{i}=A_{i} \boldsymbol{\theta}$ from (2.7), we have

$$
\hat{\boldsymbol{\beta}}_{i}=A_{i} \hat{\boldsymbol{\theta}}
$$

and

$$
\hat{\boldsymbol{\beta}}_{i, H_{j}}=A_{i} \hat{\boldsymbol{\theta}}_{j},
$$

where $\hat{\boldsymbol{\theta}}$ and $\hat{\boldsymbol{\theta}}_{j}$ are the least squares estimates of $\boldsymbol{\theta}$ under $\boldsymbol{\theta} \in \boldsymbol{\Omega}$ and under $\boldsymbol{\theta} \in \boldsymbol{\omega}_{j}$ respectively. $\hat{\boldsymbol{\theta}}$ and $\hat{\boldsymbol{\theta}}_{j}$ can be expressed as

$$
\hat{\boldsymbol{\theta}}=P \boldsymbol{y}
$$

and

$$
\hat{\boldsymbol{\theta}}_{j}=P_{j} \boldsymbol{y},
$$

where $P$ and $P_{j}$ are the orthogonal projections of $R^{n}-n$-dimensional Euclidean spaceon $\Omega$ and on $\omega_{j}$ respectively. Then

$$
\begin{gathered}
\hat{\boldsymbol{\beta}}_{i}=\hat{\boldsymbol{\beta}}_{i, H_{j}} \\
\mathbb{\mathbb { I }} \\
A_{i} P=A_{i} P_{j} \\
\mathbb{\mathbb { I }} \\
A_{i}^{\prime}=P_{j} A_{i}^{\prime} .
\end{gathered}
$$

(2.23) follows from $\Re\left[A_{i}^{\prime}\right] \subset \Omega . \quad(2.23)$ is equivalent to

$$
\Re\left[A_{i}^{\prime}\right] \subset \omega_{j} .
$$

Since $\omega_{j}=\mathfrak{N}\left[\begin{array}{c}C \\ A_{j}\end{array}\right]$ and $\Re\left[A_{i}^{\prime}\right] \perp \Re\left[C^{\prime}\right],(2.24)$ is equivalent to

$$
\Re\left[A_{i}^{\prime}\right] \perp \Re\left[A_{j}^{\prime}\right] .
$$

Then the theorem follows from Lemma 2.2. It is clear that the subscripts $i$ and $j$ are interchangeable in the proof.

q. e. d.

We can see from the proof of Theorem 2.1 that $\hat{\boldsymbol{\beta}}_{i}=\hat{\boldsymbol{\beta}}_{i, H_{j}}$ is equivalent to $\hat{\boldsymbol{\beta}}_{j}=\hat{\boldsymbol{\beta}}_{j, H_{i}}$. The same property as Theorem 2.1 has been proved by John [2] for a special situation where there are only two factors (hypotheses) in connection with the orthogonality of two factors in the design of experiments.

Theorem 2.1 gives a general result.

THEOREM 2.2. The equation

holds true if and only if

$$
\hat{\boldsymbol{\beta}}_{i}=\hat{\boldsymbol{\beta}}_{i_{, H_{j}} \cap H_{j} \cap \cdots \cap H_{j q}}
$$


PROOF. If we put

$$
H_{i} \perp H_{j_{1}}, H_{i} \perp H_{j_{2}}, \cdots, H_{i} \perp H_{j_{q}} .
$$

$$
\begin{aligned}
\boldsymbol{\omega}_{j_{1} \cdots j_{q}} & \equiv\left\{\boldsymbol{\theta} \mid \boldsymbol{\theta} \in \Omega, A_{j_{1}} \boldsymbol{\theta}=\mathbf{0}, \cdots, A_{j_{q}} \boldsymbol{\theta}=\mathbf{0}\right\} \\
& =\mathfrak{N}\left[\begin{array}{c}
C \\
A_{j_{1}} \\
\vdots \\
A_{j_{q}}
\end{array}\right],
\end{aligned}
$$

then $\hat{\boldsymbol{\beta}}_{i_{1, H_{j_{1}} \cap \cdots \cap H_{j q}}}$ is the least squares estimate of $\boldsymbol{\beta}_{i}$ under $\boldsymbol{\theta} \in \omega_{j_{1} \cdots j_{q}}$. Since $\boldsymbol{\beta}_{i}=A_{i} \boldsymbol{\theta}$, we have

$$
\hat{\boldsymbol{\beta}}_{i, H_{j_{1}} \cap \cdots \cap H_{j_{q}}}=A_{i} \hat{\boldsymbol{\theta}}_{j_{1} \cdots j_{q}},
$$

where $\hat{\boldsymbol{\theta}}_{j_{1} \cdots j_{q}}$ is the least squares estimate of $\boldsymbol{\theta}$ under $\boldsymbol{\theta} \in \omega_{j_{1} \cdots j_{q}}$. Since $\hat{\boldsymbol{\theta}}_{j_{1} \cdots j_{q}}$ can be expressed by $\hat{\boldsymbol{\theta}}_{j_{1} \cdots j_{q}}=P_{j_{1} \cdots j_{q}} \boldsymbol{y}$, (2.27) turns out to be

$$
\hat{\boldsymbol{\beta}}_{i, H_{j_{1}} \cap \cdots \cap H_{j_{q}}}=A_{i} P_{j_{1} \cdots j_{q}} \boldsymbol{y},
$$

where $P_{j_{1} \cdots j_{q}}$ is the orthogonal projection of $R^{n}$ on $\omega_{j_{1} \cdots j_{q}}$.

Since $\hat{\boldsymbol{\beta}}_{i}=A_{i} P \boldsymbol{y}$, similarly to the proof of Theorem 2.1, we have the following equivalence relations :

(2.30) is equivalent to

$$
\begin{gathered}
\hat{\boldsymbol{\beta}}_{i}=\hat{\boldsymbol{\beta}}_{i, H_{j_{1}} \cap \cdots \cap H_{j_{q}}} \\
\mathbb{I} \\
A_{i} P=A_{i} P_{j_{1} \cdots j_{q}} \\
\mathbb{\mathbb { }} \\
A_{i}^{\prime}=P_{j_{1} \cdots j_{q}} A_{i}^{\prime} \\
\mathbb{I} \\
\Re\left[A_{i}^{\prime}\right] \subset \omega_{j_{1} \cdots j_{q}} .
\end{gathered}
$$

$$
\Re\left[A_{i}^{\prime}\right] \perp \Re\left[A_{j_{1}}^{\prime}\right], \cdots, \Re\left[A_{i}^{\prime}\right] \perp \Re\left[A_{j_{q}}^{\prime}\right],
$$

because $\mathfrak{R}\left[A_{i}^{\prime}\right] \perp \mathfrak{R}\left[C^{\prime}\right]$. Then, the theorem follows from Lemma 2.2 .

q. e. d.

From Theorem 2.1 and Theorem 2.2, we have the following corollary.

COROLLARY. $H_{j_{1}}, H_{j_{2}}, \cdots, H_{j_{q}}$ are orthogonal if and only if the least squares estimates of $\boldsymbol{\beta}_{j_{1}}$ under the model (1.1), under $H_{j_{2}}, \cdots$, under $H_{j_{q}}$, under $H_{j_{2}} \cap H_{j_{3}}, \cdots$, and under $H_{j_{2}} \cap \cdots \cap H_{j_{q}}$ are the same and this property also holds true for other parameter vectors $\boldsymbol{\beta}_{j_{2}}, \cdots, \boldsymbol{\beta}_{j_{q}}$.

THEOREM 2.3. Suppose that $H_{l} \perp H_{j_{1}}, \cdots, H_{l} \perp H_{j_{q}}$. Then

$$
H_{i} \perp H_{l} \Leftrightarrow \hat{\boldsymbol{\beta}}_{i_{i, H_{1}} \cap \cdots \cap H_{j_{q}}}=\hat{\boldsymbol{\beta}}_{i_{i, H} \cap \cdots \cap H_{j_{q}} \wedge H_{l}} .
$$

PROOF. Let $\omega_{j_{1} \cdots j_{q}}$ and $\omega_{j_{1} \cdots j_{q_{q}}}$ be two subspaces such that

$$
\begin{aligned}
\omega_{j_{1} \cdots j_{q}} & =\left\{\boldsymbol{\theta} \mid \boldsymbol{\theta} \in \Omega, A_{j_{1}} \boldsymbol{\theta}=\mathbf{0}, \cdots, A_{j_{q}} \boldsymbol{\theta}=\mathbf{0}\right\} \\
& =\Re\left[\begin{array}{c}
C \\
A_{j_{1}} \\
\vdots \\
A_{j_{q}}
\end{array}\right],
\end{aligned}
$$




$$
\begin{aligned}
\omega_{j_{1} \cdots q_{q} l} & =\left\{\boldsymbol{\theta} \mid \boldsymbol{\theta} \in \Omega, A_{j_{1}} \boldsymbol{\theta}=\mathbf{0}, \cdots, A_{j_{q}} \boldsymbol{\theta}=\mathbf{0} \text { and } A_{l} \theta=\mathbf{0}\right\} \\
& =\Re\left[\begin{array}{c}
C \\
A_{j_{1}} \\
\vdots \\
A_{j_{q}} \\
\hline A_{l}
\end{array}\right]
\end{aligned}
$$

and let $P_{j_{1} \cdots j_{q}}$ and $P_{j_{1} \cdots j_{q} l}$ be the orthogonal projections of $R^{n}$ on $\omega_{j_{1} \cdots j_{q}}$ and on $\omega_{j_{1} \cdots j_{q} l}$ respectively.

As in the proof of Theorem 2.2, we have

and

$$
\hat{\boldsymbol{\beta}}_{i, H_{j_{1}} \cap \cdots \cap H_{j_{q}}}=A_{i} P_{j_{1} \cdots j_{q}} \boldsymbol{y}
$$

Then

$$
\hat{\boldsymbol{\beta}}_{i, H_{j_{1} \cap \cdots \cap H_{j} \cap H_{l}}}=A_{i} P_{j_{1} \cdots j_{q}} l \boldsymbol{y} .
$$

$$
\begin{gathered}
\hat{\boldsymbol{\beta}}_{i_{,}, H_{j_{1}} \cap \cdots \cap H_{j_{q}}}=\hat{\boldsymbol{\beta}}_{i, H_{j_{1}} \cap \cdots \cap H_{j_{q}} \cap H_{l}} \\
\hat{\mathbb{I}} \\
\left(P_{j_{1} \cdots j_{q}}-P_{j_{1} \cdots j_{q} l}\right) A_{i}^{\prime}=0 .
\end{gathered}
$$

It is easily shown that $P_{j_{1} \cdots j_{q}}-P_{j_{1} \cdots j_{q}}$ is the orthogonal projection of $R^{n}$ on $\omega_{j_{1} \cdots j_{q}} \cap\left(\omega_{j_{1} \cdots j_{q}}\right)^{\perp}$. Therefore, (2.33) means that all the column vectors of the matrix $A_{i}^{\prime}$ belong to the orthogonal complement of $\omega_{j_{1} \cdots j_{q}} \cap\left(\omega_{j_{1} \cdots j_{q}}\right)^{\perp}$. Thus (2.33) is equivalent to

$$
\Re\left[A_{i}^{\prime}\right] \subset\left(\omega_{j_{1} \cdots j_{q}} \cap\left(\omega_{j_{1} \cdots j_{q}} l\right)^{\perp}\right)^{\perp} .
$$

By the assumption of the theorem and Lemma 2.3, (2.34) turns out to be

which is equivalent to

$$
\Re\left[A_{i}^{\prime}\right] \subset\left(\Re\left[A_{l}^{\prime}\right]\right)^{\perp},
$$

$$
\Re\left[A_{i}^{\prime}\right] \perp \Re\left[A_{l}^{\prime}\right] .
$$

Thus the theorem follows by Lemma 2.2 .

q. e. d.

Before concluding this section, we shall give simple proofs of two well-known and important results about the orthogonality property by using the null space representation for the hypotheses.

(1) Definitions 1.1 and 1.2 on the orthogonality of hypotheses are equivalent.

PRoOF.

$$
\begin{aligned}
\operatorname{cov}\left(\hat{\boldsymbol{\beta}}_{i}, \hat{\boldsymbol{\beta}}_{j}\right) & =\operatorname{cov}\left(A_{i} \hat{\boldsymbol{\theta}}, A_{j} \hat{\boldsymbol{\theta}}\right) \\
& =\operatorname{cov}\left(A_{i} P \boldsymbol{y}, A_{j} P \boldsymbol{y}\right)=A_{i} A_{j}^{\prime} \boldsymbol{\sigma}^{2} .
\end{aligned}
$$

Hence

$$
\operatorname{cov}\left(\hat{\boldsymbol{\beta}}_{i}, \hat{\boldsymbol{\beta}}_{j}\right)=0 \Leftrightarrow \Re\left[A_{i}^{\prime}\right] \perp \Re\left[A_{j}^{\prime}\right] .
$$

Then the result follows from Lemma 2.1 .

q. e. d.

(2) The sum of squares for the hypothesis $H_{i}, Q_{i}$, is the same as the one, $Q_{i ; 1,2, \ldots, i-1}$, calculated under $H_{1} \cap H_{2} \cap \cdots \cap H_{i-1}$ if and only if

$$
H_{i} \perp H_{1}, H_{i} \perp H_{2}, \cdots, H_{i} \perp H_{i-1} .
$$


PROOF.

and

$$
Q_{i}=\boldsymbol{y}^{\prime}\left(P-P_{i}\right) \boldsymbol{y}
$$

$$
Q_{i ; 1,2, \cdots, i-1}=\boldsymbol{y}^{\prime}\left(P_{12 \cdots i-1}-P_{12 \cdots i}\right) \boldsymbol{y},
$$

where $P, P_{i}, P_{12 \cdots i-1}, P_{12 \cdots i}$ are the orthogonal projections of $R^{n}$ on $\Omega$, on $\omega_{i}$, on $\omega_{12 \cdots i-1}$ and on $\omega_{12 \cdots i}$ respectively, $\omega_{12 \cdots i-1}$ and $\omega_{12 \cdots i}$ being defined as in the proof of Theorem 2.2.

Since $P-P_{i}$ and $P_{12 \cdots i-1}-P_{12 \cdots i}$ are the orthogonal projections of $R^{n}$ on $\Omega \cap \omega_{i}^{\frac{1}{}}$ and on $\omega_{12 \cdots i-1} \cap\left(\omega_{12 \cdots i}\right)^{\perp}, Q_{i}=Q_{i ; 12 \cdots i-1}$ is equivalent to

$$
\omega_{12 \cdots i-1} \cap\left(\omega_{12 \cdots i}\right)^{\perp}=\Omega \cap \omega_{i}^{\perp} .
$$

From Lemma 2.1 and Lemma 2.3, (2.37) is equivalent to

and the result is proved.

$$
H_{i} \perp H_{1}, H_{i} \perp H_{2}, \cdots, H_{i} \perp H_{i-1},
$$

\section{Identifiability Constraints and the Orthogonality of Hypotheses in the} Analysis of Variance Model

The sum of squares for the hypothesis $H_{i}, Q_{i}$, is given by

$$
Q_{i}=\boldsymbol{y}^{\prime}\left(P-P_{i}\right) \boldsymbol{y}=\left(\hat{\boldsymbol{\theta}}-\hat{\boldsymbol{\theta}}_{i}\right)^{\prime}\left(\hat{\boldsymbol{\theta}}-\hat{\boldsymbol{\theta}}_{i}\right) .
$$

Since $\hat{\boldsymbol{\theta}}=X \hat{\boldsymbol{\beta}}=\sum_{j} X_{j} \hat{\boldsymbol{\beta}}_{j}$, we can see from Theorem 2.1 that if $H_{i}$ is orthogonal to every other hypotheses $H_{1}, \cdots, H_{i-1}, H_{i+1}, \cdots, H_{k}$ then $Q_{i}$ can be calculated by

$$
Q_{i}=\left(X_{i} \hat{\boldsymbol{\beta}}_{i}\right)^{\prime}\left(X_{i} \hat{\boldsymbol{\beta}}_{i}\right)
$$

or

$$
Q_{i}=\hat{\boldsymbol{\beta}}_{i}^{\prime}\left(X_{i}^{\prime} \boldsymbol{y}\right),
$$

using the least squares estimate $\hat{\boldsymbol{\beta}}_{i}$ of $\boldsymbol{\beta}_{i}$ under $\boldsymbol{\theta} \in \boldsymbol{Q}$ without calculating the least squares estimates of $\boldsymbol{\beta}_{j}, j=1,2, \cdots, i-1, i+1, \cdots, k$, under $\boldsymbol{\theta} \in \boldsymbol{\omega}_{i}$.

Therefore, in order to make the calculation of the sums of squares for hypotheses simpler, it is needed that each of the hypotheses of interest is orthogonal to every other hypotheses.

Unfortunately, since orthogonality property of hypotheses depends on identifiability constraints chosen in the analysis of variance model, it is desirable to choose suitable ones for orthogonality property.

In discussing the relationship between identifiability constraints and orthogonality of hypotheses, we put the following assumptions on the design matrix and identifiability constraints according to the analysis of variance model.

Assumptions (i) $X_{1}$ is an $n \times 1$ vector consisting solely of 1 . Then $\beta_{1}$ is a scalar called general mean.

(ii) Identifiability constraints have the form such that

$$
B_{2} \boldsymbol{\beta}_{2}=\mathbf{0}, \cdots, B_{k} \boldsymbol{\beta}_{k}=\mathbf{0},
$$

where $B_{i}, i=2,3, \cdots, k$, are $t_{i} \times p_{i}$ matrices, i. e. identifiability constraints are given 
within each of the parameter groups $\boldsymbol{\beta}_{i}, i=2,3, \cdots, k$ except $\beta_{1}$.

The normal equations for obtaining the least squares estimates $\hat{\beta}_{1}, \hat{\boldsymbol{\beta}}_{2}, \cdots, \hat{\boldsymbol{\beta}}_{k}$ of $\boldsymbol{\beta}_{1}, \boldsymbol{\beta}_{2}, \cdots, \boldsymbol{\beta}_{k}$ are given by

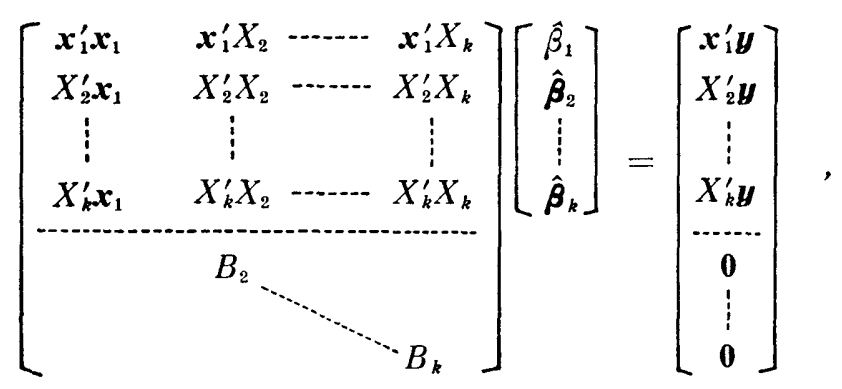

which are arranged in a table as (3.6).

\begin{tabular}{cccc|c}
\hline$\hat{\boldsymbol{\beta}}_{1}^{\prime}$ & $\hat{\boldsymbol{\beta}}_{2}^{\prime}$ & $\cdots$ & $\hat{\boldsymbol{\beta}}_{k}^{\prime}$ & $=1$ \\
\hline $\boldsymbol{x}_{1}^{\prime} \boldsymbol{x}_{1}$ & $\boldsymbol{x}_{1}^{\prime} X_{2}$ & $\cdots$ & $\boldsymbol{x}_{1}^{\prime} X_{k}$ & $\boldsymbol{x}_{1}^{\prime} \boldsymbol{y}$ \\
$X_{2}^{\prime} \boldsymbol{x}_{1}$ & $X_{2}^{\prime} X_{2}$ & $\cdots$ & $X_{2}^{\prime} X_{k}$ & $X_{2}^{\prime} \boldsymbol{y}$ \\
$\vdots$ & $\vdots$ & & $\vdots$ & $\vdots$ \\
$X_{k}^{\prime} \boldsymbol{x}_{1}$ & $X_{k}^{\prime} X_{2}$ & $\cdots$ & $X_{k}^{\prime} X_{k}$ & $X_{k}^{\prime} \boldsymbol{y}$ \\
\hline & $B_{2}$ & & & $\mathbf{0}$ \\
& & $\ddots$ & & $\vdots$ \\
& & $\ddots$ & $B_{k}$ & $\mathbf{0}$ \\
\hline
\end{tabular}

It is known that all the Lagrange multipliers corresponding to the identifiability constraints can be ignored in the normal equations (See, for example, Washio [6]). It is also known that the normal equations (3.6) have a unique solution for every $\boldsymbol{y}$.

THEOREM 3.1. Suppose that the design matrix and identifiability constraints satisfy the assumptions given just before. Suppose further that the identifiability constraints satisfy the following additional assumption: In a model

$$
\boldsymbol{y}=\left[\begin{array}{lllll}
\boldsymbol{x}_{1} & X_{2} & \cdots & X_{i-1}
\end{array}\right]\left[\begin{array}{c}
\beta_{1} \\
\boldsymbol{\beta}_{2} \\
\vdots \\
\boldsymbol{\beta}_{i-1}
\end{array}\right]+\boldsymbol{e}
$$

which is the model that $H_{k}, H_{k-1}, \cdots, H_{i}$ are assumed to be true, the constraints

$$
B_{2} \beta_{2}=0, \cdots, B_{i-1} \beta_{i-1}=0
$$

are still identifiability constraints for the new model (3.7) and this property holds true for $i=k, k-1, \cdots, 3$.

Then, all the hypotheses $H_{1}, H_{2}, \cdots, H_{k}$ are orthogonal if and only if all the elements above the diagonal blocks in the left-hand side in the normal equations (3.6) are crossed 
off by using identifiability constraints, i.e. the normal equations (3.6) turn out to be of the form:

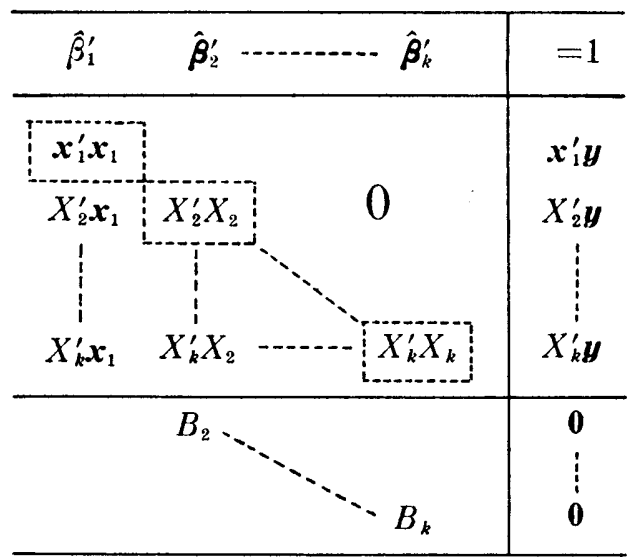

Proof. Suppose the normal equations are of the form in (3.9). The normal equations for obtaining the least squares estimates of $\boldsymbol{\beta}_{1}, \boldsymbol{\beta}_{2}, \cdots, \boldsymbol{\beta}_{k-1}$ when $H_{k}$ is true (under $H_{k}$ ) are given by

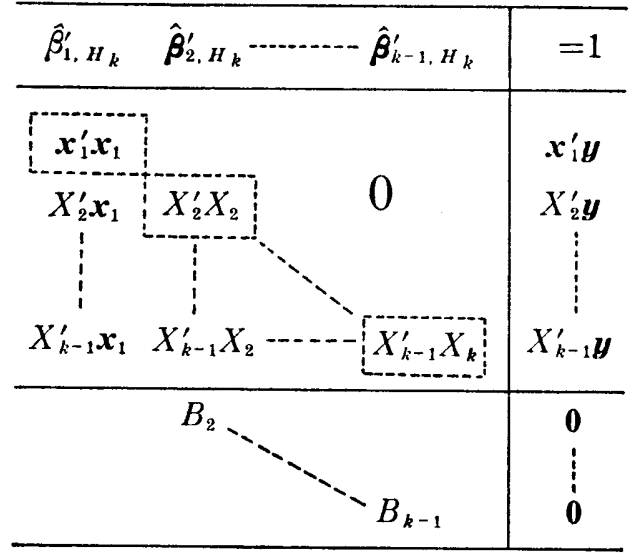

since the Lagrange multipliers corresponding to $B_{2} \boldsymbol{\beta}_{2, H_{k}}=\mathbf{0}, \cdots, B_{k-1} \boldsymbol{\beta}_{k-1, H_{k}}=\mathbf{0}$ can be ignored by the assumption of the theorem. By comparing (3.9) with (3.10), we get

which show

$$
\hat{\beta}_{1}=\hat{\beta}_{1, H_{k}}, \cdots, \hat{\boldsymbol{\beta}}_{k-1}=\hat{\boldsymbol{\beta}}_{k-1, H_{k}},
$$

by Theorem 2.1.

$$
H_{k} \perp H_{1}, H_{k} \perp H_{2}, \cdots, H_{k} \perp H_{k-1}
$$

Similarly, for the least squares estimates of $\beta_{1}, \boldsymbol{\beta}_{2}, \cdots, \boldsymbol{\beta}_{k-2}$ under $H_{k}$ and under $H_{k} \cap H_{k-1}$, we can see

which show

$$
\hat{\beta}_{1, H_{k}}=\hat{\boldsymbol{\beta}}_{1, H_{k} \cap H_{k-1}}, \cdots, \hat{\boldsymbol{\beta}}_{k-2, H_{k}}=\hat{\boldsymbol{\beta}}_{k-2, H_{k} \cap H_{k-1}},
$$

$$
H_{k-1} \perp H_{1}, H_{k-1} \perp H_{2}, \cdots, H_{k-1} \perp H_{k-2}
$$


by Theorem 2.3. Similarly, for the least squares estimates of $\boldsymbol{\beta}_{1}, \boldsymbol{\beta}_{2}, \cdots, \boldsymbol{\beta}_{k-3}$ under $H_{k} \cap H_{k-1}$ and under $H_{k} \cap H_{k-1} \cap H_{k-2}$, we can see

which show

$$
\begin{gathered}
\hat{\beta}_{1, H_{k} \cap H_{k-1}}=\hat{\boldsymbol{\beta}}_{1, H_{k} \cap H_{k-1} \cap H_{k-2}} \\
\vdots \\
\hat{\boldsymbol{\beta}}_{k-3, H_{k} \cap H_{k-1}}=\hat{\boldsymbol{\beta}}_{k-3, H_{k} \cap H_{k-1} \cap H_{k-2}},
\end{gathered}
$$

by Theorem 2.3.

$$
H_{k-2} \perp H_{1}, H_{k-2} \perp H_{2}, \cdots, H_{k-2} \perp H_{k-3}
$$

By repeating the same procedure, we can show all the hypotheses are orthogonal.

Conversely, suppose $H_{1}, H_{2}, \cdots, H_{k}$ are orthogonal. From the corollary in Section 2 , the least squares estimates of $\boldsymbol{\beta}_{i}$ 's under the model (1.1), under $H_{k}$, under $H_{k} \cap H_{k-1}, \cdots$, under $H_{k} \cap \cdots \cap H_{2}$ are all the same. Thus by considering normal equations for obtaining the least squares estimates of $\boldsymbol{\beta}_{i}$ 's under the various models just mentioned above, we have

$$
\begin{array}{cccc}
\boldsymbol{x}_{1}^{\prime} X_{2} \hat{\boldsymbol{\beta}}_{2}=0 \quad \boldsymbol{x}_{1}^{\prime} X_{3} \hat{\boldsymbol{\beta}}_{3}=0 & \cdots & \boldsymbol{x}_{1}^{\prime} X_{k} \hat{\boldsymbol{\beta}}_{k}=0 \\
& X_{2}^{\prime} X_{3} \hat{\boldsymbol{\beta}}_{3}=0 & \cdots & X_{2}^{\prime} X_{k} \hat{\boldsymbol{\beta}}_{k}=0 \\
& & \ddots & \vdots \\
& & \ddots & \vdots \\
& & & X_{k-1}^{\prime} X_{k} \hat{\boldsymbol{\beta}}_{k}=0
\end{array}
$$

for every $\boldsymbol{y}$, where $\hat{\boldsymbol{\beta}}_{i}$ 's are the least squares estimates under the model (1.1).

Since $\hat{\beta}_{1}$ and $\hat{\boldsymbol{\beta}}_{2}$ are the unique solution to the equations

$$
\left[\begin{array}{cc}
x_{1}^{\prime} x_{1} & x_{1}^{\prime} X_{2} \\
X_{2}^{\prime} x_{1} & X_{2}^{\prime} X_{2} \\
0 & B_{2}
\end{array}\right]\left[\begin{array}{c}
\hat{\beta}_{1} \\
\hat{\beta}_{2}
\end{array}\right]=\left[\begin{array}{c}
x_{1}^{\prime} y \\
X_{2}^{\prime} y \\
0
\end{array}\right],
$$

we can show that the equations $\boldsymbol{x}_{1}^{\prime} X_{2} \hat{\boldsymbol{\beta}}_{2}=0$ should be generated by the equation (3.12), and therefore, should be generated by $B_{2} \hat{\boldsymbol{\beta}}_{2}=0$. Thus the cell of $\boldsymbol{x}_{1}^{\prime} X_{2}$ can be crossed off by the identifiability constraints.

Similarly, since $\hat{\beta}_{1}, \hat{\boldsymbol{\beta}}_{2}$ and $\hat{\boldsymbol{\beta}}_{3}$ are also the unique solution to the equations

$$
\left[\begin{array}{ccc}
\boldsymbol{x}_{1}^{\prime} \boldsymbol{x}_{1} & \boldsymbol{x}_{1}^{\prime} X_{2} & \boldsymbol{x}_{1}^{\prime} X_{3} \\
X_{2}^{\prime} \boldsymbol{x}_{1} & X_{2}^{\prime} X_{2} & X_{2}^{\prime} X_{3} \\
X_{3}^{\prime} \boldsymbol{x}_{1} & X_{3}^{\prime} X_{2} & X_{3}^{\prime} X_{3} \\
0 & B_{2} & 0 \\
0 & 0 & B_{3}
\end{array}\right]\left[\begin{array}{c}
\hat{\beta}_{1} \\
\hat{\boldsymbol{\beta}}_{2} \\
\hat{\boldsymbol{\beta}}_{3}
\end{array}\right]=\left[\begin{array}{c}
\boldsymbol{x}_{1}^{\prime} \boldsymbol{y} \\
X_{2}^{\prime} \boldsymbol{y} \\
X_{3}^{\prime} \boldsymbol{y} \\
\mathbf{0} \\
\mathbf{0}
\end{array}\right],
$$

then $\boldsymbol{x}_{1}^{\prime} X_{3} \hat{\boldsymbol{\beta}}_{3}=0$ and $X_{2}^{\prime} X_{3} \hat{\boldsymbol{\beta}}_{3}=\mathbf{0}$ should be generated by the equations (3.13), and then should be generated by $B_{3} \hat{\boldsymbol{\beta}}_{3}=\mathbf{0}$. Thus the cells of $\boldsymbol{x}_{1}^{\prime} X_{3}$ and $X_{2}^{\prime} X_{3}$ can be crossed off by the identifiability constraints.

By repeating the same procedure, all the cells above the diagonal ones can be crossed off. Thus the theorem is proved.

q. e. d.

Although the additional assumption on identifiability constraints in Theorem 3.1 
seems tight, it will be satisfied by most of analysis of variance models by arranging the parameters in proper order. Therefore, Theorem 3.1 will provide us with an easy and simple method for finding the identifiability constraints which make all the hypotheses orthogonal.

The method proposed by Theorem 3.1 is illustrated with two models.

EXAMPLE 1. The two-way layout with one observation per cell.

Consider a two-way layout with $I$ levels of factor $A, J$ levels of factor $B$ and one observation per cell. Let $y_{i j}$ be the observation in the $\left(A_{i}, B_{j}\right)$ cell, and assume the model

$$
y_{i j}=\mu+\alpha_{i}+\beta_{j}+e_{i j}, \quad i=1,2, \cdots, I ; \quad j=1,2, \cdots, J
$$

where $\mu$ represents the general mean, $\alpha_{i}$ the main effect of factor $A, \beta_{j}$ main effect of factor $B$ and $e_{i j}$ the random error.

As the identifiability constraints are given within each of the parameter groups, the class of identifiability constraints is given by

$$
\sum_{i} u_{i} \alpha_{i}=0, \quad \sum_{j} v_{j} \beta_{j}=0
$$

where $\sum_{i} u_{i}=1$ and $\sum_{j} v_{j}=1$. It is easy to see that the assumptions in Theorem 3.1 are all satisfied by this model having identifiability constraints (3.15).

Since the left-hand side in the normal equations for obtaining the least squares estimates of parameters is given by Table 1 ,

Table 1

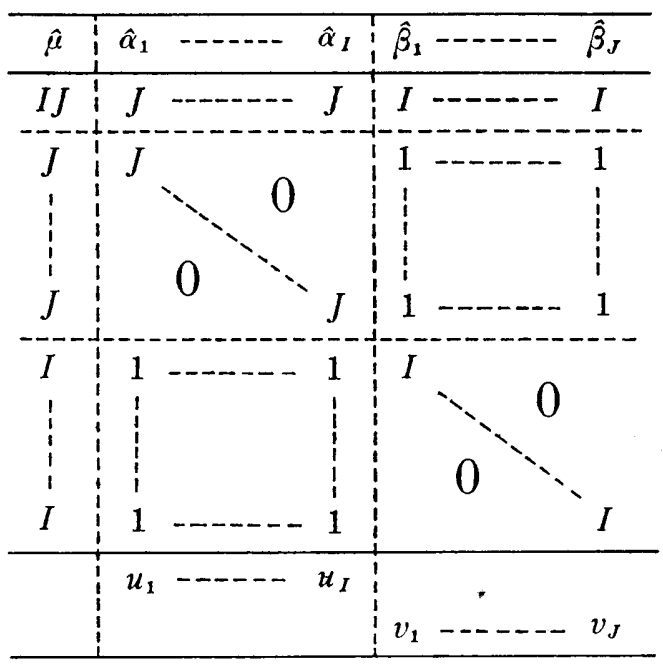

we can conclude from Theorem 3.1 that $H_{1}(\mu=0), H_{2}\left(\alpha_{1}=\cdots=\alpha_{I}=0\right)$ and $H_{3}\left(\beta_{1}=\cdots\right.$ $=\beta_{J}=0$ ) are orthogonal if and only if 


$$
\begin{aligned}
& u_{1}=u_{2}=\cdots=u_{I}=\frac{1}{I} \\
& v_{1}=v_{2}=\cdots=v_{J}=\frac{1}{J} .
\end{aligned}
$$

This result coincides with that of Seber [5].

EXAMPLE 2. The two-way layout with unequal numbers of observations in the cells.

Consider a two-way layout with $I$ levels of factor $A, J$ levels of factor $B$ and $n_{i j}$ observations in the $\left(A_{i}, B_{j}\right)$ cell. If $y_{i j k}$ denotes the $k$-th observation in the $\left(A_{i}, B_{j}\right)$ cell, then $y_{i j k}$ can be written as

$$
\begin{aligned}
& y_{i j k}=\mu+\alpha_{i}+\beta_{j}+\gamma_{i j}+e_{i j k}, \\
& \quad i=1,2, \cdots, I ; j=1,2, \cdots, J ; k=1,2, \cdots, n_{i j},
\end{aligned}
$$

where $\mu, \alpha_{i}$ and $\beta_{j}$ are as in Example $1, \gamma_{i j}$ the interaction between $A$ and $B$ and $e_{i j k}$ the random error.

As a class of identifiability constraints, we shall consider

$$
\begin{array}{ll}
\sum_{i} u_{i} \alpha_{i}=0, & \sum_{j} v_{j} \beta_{j}=0, \\
\sum_{i} u_{i} \gamma_{i j}=0, & \text { for all } j, \\
\sum_{j} v_{j} \gamma_{i j}=0, & \text { for all } i,
\end{array}
$$

where $\sum_{i} u_{i}=1$ and $\sum_{j} v_{j}=1$.

It is easy to see that the assumptions in Theorem 3.1 are all satisfied by this model having identifiability constraints (3.18). In this example, the left-hand side in the normal equations is given by Table 2 .

In order to cross off all the cells above the diagonal ones by using identifiability constraints, it is necessary that

$$
\begin{array}{ll}
\text { (i) } n_{i}=Q u_{i}, & \text { for all } i \\
\text { (ii) } n_{\cdot j}=R v_{j}, & \text { for all } j \\
\text { (iii) } n_{i j}=S_{i} v_{j}, & \text { for all } i, j \\
\text { (iv) } n_{i j}=T_{j} u_{i}, & \text { for all } i, j,
\end{array}
$$

where $Q, R, S_{i}$ and $T_{j}$ are some constants. From (i), (ii) and (iii) in (3.19), we have

$$
n_{. .}=Q, \quad n_{. .}=R, \quad n_{i}=S_{i}
$$

because of $\sum_{i} u_{i}=1$ and $\sum_{j} v_{j}=1$. Then, again from (i), (ii) and (iii), we have

$$
\begin{array}{ll}
u_{i}=\frac{n_{i \cdot}}{n . .}, & \text { for all } i \\
v_{j}=\frac{n . j_{\cdot j}}{n . .}, & \text { for all } j \\
n_{i j}=\frac{n_{i \cdot n \cdot j}}{n . .} & \text { for all } i, j .
\end{array}
$$


Table 2

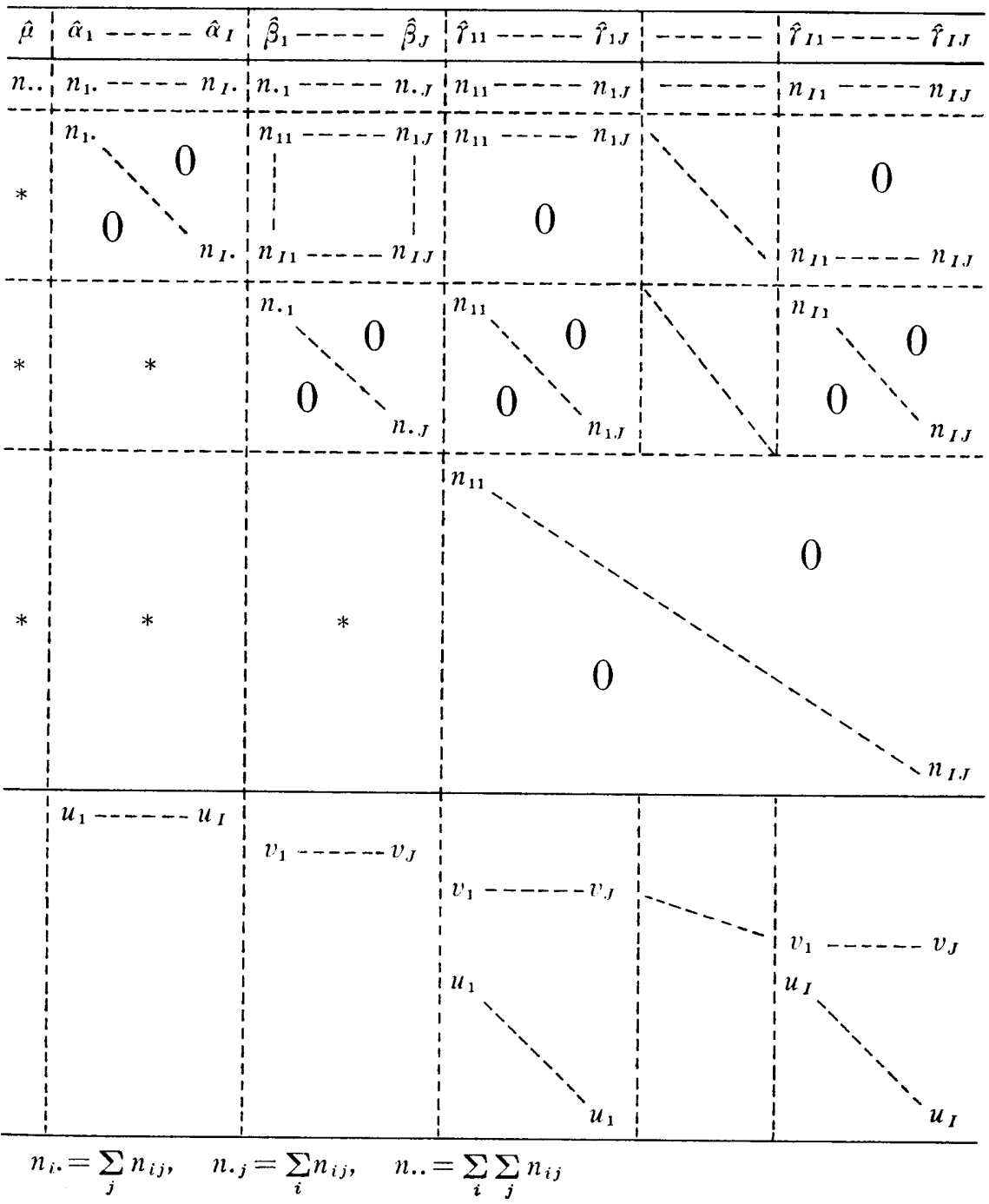

We can easily check that if $u_{i}$ 's, $v_{j}$ 's and $n_{i j}$ 's are as in (3.20) then all the cells above the diagonal ones are crossed off by using identifiability constraints (3.18). Then, (3.20) is the necessary and sufficient condition for the four hypotheses $H_{1}, H_{2}, H_{3}, H_{4}$ $\left(\gamma_{11}=\gamma_{12}=\cdots=\gamma_{I J}=0\right)$ to be orthogonal.

Seber [5] obtained the condition (3.20) under the further assumption of $u_{i} \geqq 0$ and $v_{j} \geqq 0$.

Although even if we relax the class of identifiability constraints to

$$
\begin{array}{ll}
\sum_{i} u_{i} \alpha_{i}=0, & \sum_{j} v_{j} \beta_{j}=0 \\
\sum_{i} l_{i} \gamma_{i j}=0, & \text { for all } j \\
\sum_{j} m_{j} \gamma_{i j}=0, & \text { for all } i,
\end{array}
$$


where $\sum_{i} u_{i}=1, \sum_{j} v_{j}=1, \sum_{i} l_{i}=1$ and $\sum_{j} m_{j}=1$, we can get the similar necessary and sufficient condition, we have not checked if (3.21) is identifiability constraint.

\section{References}

[1] Darroch, J.N. and Silvey, S.D.: On testing more than one hypothesis, Ann. Math. Stat., 34 (1963), 555-567.

[2] JoHN, P.W.M.: Statistical design and analysis of experiments, The Macmillan. (1971).

[3] KEMPTHORNE, O.: The design and analysis of experiments, Wiley. (1952).

[4] SCHEFFE, H.: The analysis of variance, Wiley. 1959.

[5] SEBER, G.A.F.: The linear hypothesis, Griffin. (1966).

[6] Washio, Y.: On Lagrange multipliers in normal equations, Proc. Fujihara Men. Fac. Engin. Keio Univ., 23 (1970), 87-99.

Communicated by T. Kilagawa

Received October 27, 1984 